\title{
Quantitative Reasoning Learning Progressions for Environmental Science: Developing a Framework
}

\author{
Robert L. Mayes \\ Georgia Southern University, rmayes@georgiasouthern.edu \\ Franziska Peterson \\ University of Wyoming \\ Rachel Bonilla \\ Georgia Southern University
}

Follow this and additional works at: https://digitalcommons.usf.edu/numeracy

Part of the Educational Assessment, Evaluation, and Research Commons, and the Science and Mathematics Education Commons

\section{Recommended Citation}

Mayes, Robert L., Franziska Peterson, and Rachel Bonilla. "Quantitative Reasoning Learning Progressions for Environmental Science: Developing a Framework." Numeracy 6, Iss. 1 (2013): Article 4. DOI:

http://dx.doi.org/10.5038/1936-4660.6.1.4 


\title{
Quantitative Reasoning Learning Progressions for Environmental Science: Developing a Framework
}

\begin{abstract}
Quantitative reasoning is a complex concept with many definitions and a diverse account in the literature. The purpose of this article is to establish a working definition of quantitative reasoning within the context of science, construct a quantitative reasoning framework, and summarize research on key components in that framework. Context underlies all quantitative reasoning; for this review, environmental science serves as the context.In the framework, we identify four components of quantitative reasoning: the quantification act, quantitative literacy, quantitative interpretation of a model, and quantitative modeling. Within each of these components, the framework provides elements that comprise the four components. The quantification act includes the elements of variable identification, communication, context, and variation. Quantitative literacy includes the elements of numeracy, measurement, proportional reasoning, and basic probability/statistics. Quantitative interpretation includes the elements of representations, science diagrams, statistics and probability, and logarithmic scales. Quantitative modeling includes the elements of logic, problem solving, modeling, and inference. A brief comparison of the quantitative reasoning framework with the AAC\&U Quantitative Literacy VALUE rubric is presented, demonstrating a mapping of the components and illustrating differences in structure. The framework serves as a precursor for a quantitative reasoning learning progression which is currently under development.
\end{abstract}

\section{Keywords}

quantitative reasoning, learning progression, quantification, quantitative literacy, modeling

\section{Creative Commons License}

\section{()) (9)}

This work is licensed under a Creative Commons Attribution-Noncommercial 4.0 License

\section{Cover Page Footnote}

Robert Mayes is a Research Professor in Education at Georgia Southern University and Director of the Institute for Interdisciplinary STEM Education (i2 STEMe). His research focus is on quantitative reasoning in STEM and development of a QR learning progression within the context of science. His work supports interdisciplinary STEM teaching that impacts underrepresented populations and rural areas.

Franziska Peterson is a doctoral student in mathematics education at the University of Wyoming. She is researching QR and learning progressions as part of a project supported partially by a grant from the NSF: Culturally Relevant Ecology, Learning Progressions, and Environmental Literacy (DUE-0832173). Her additional research interest focuses on the role of language in $Q R$.

Rachel Bonilla is a mathematics and statistics graduate student in the Department of Mathematical Sciences and is a research assistant in the Department of Teaching and Learning at Georgia Southern University. She is working in part with the QR Theme Team of the DUE-0832173 project. Her research interests also include statistical literacy in K-12 students. 


\section{Introduction}

The research goal of the NSF project, "Culturally Relevant Ecology, Learning Progressions, and Environmental Literacy" is to refine and extend current frameworks and assessments for learning progressions that lead to environmental science literacy and associated mathematics. A central component of the project is to develop learning progressions that provide a trajectory along which middle and high school students become environmentally literate citizens. The project has produced learning progressions with supporting assessments and teaching experiments in three areas: carbon cycle and storage; water cycle and transport; and biodiversity communities and extinction. The progressions have been verified through research conducted in collaboration with Long Term Ecological Research Sites (LTERS) and partner school districts in Colorado, California, Michigan, and Maryland.

Why learning progressions? The Consortium for Policy Research in Education (CPRE) report, Learning Progressions in Science: An Evidence-based Approach to Reform (Corcoran et al. 2009), identified learning progressions as a promising model that can advance effective adaptive-instruction teaching techniques and thereby change the norms of practice in schools. The CPRE report defines a learning progression as a set of "empirically grounded and testable hypotheses about how students' understanding of, and ability to use, core scientific concepts and explanations and related scientific practices grow and become more sophisticated over time, with appropriate instruction" (Corcoran et al. 2009, p. 8). Learning progressions in environmental literacy support a broad public consensus about the value of understanding ecosystem functions, even though the public demonstrates a poor understanding of ecosystems.. The progressions aim to address the pressing real-world need to understand the consequences of current actions on the future of that ecosystem.

Why quantitative reasoning? The project consists of multiple research teams including a carbon strand, a water strand, and a biodiversity strand, as well as supporting research themes for citizenship and quantitative reasoning $(\mathrm{QR})$. The science strands incorporate socio-ecological systems associated with human impact on the environment. This requires understanding principles of scale; tracing matter and energy through a system; sustainability over time; making scientifically informed decisions; precision in measurement and description; and changing nature of accounts from narrative reasoning to model-based reasoning. All of these have quantitative aspects that make $\mathrm{QR}$ essential to advancing

\footnotetext{
${ }^{1}$ This project is supported in part by a grant from the National Science Foundation: Culturally Relevant Ecology, Learning Progressions, and Environmental Literacy (DUE-0832173) which we refer to as Pathways.
} 
environmental literacy. For example, informed citizens must move from a macroscopic scale to view environmental issues at atomic-molecular and microscopic scales that explain hidden mechanisms. Moving to these scales engages students in the inherently quantitative sciences of chemistry and physics. In addition, citizens must generalize from the local to landscape/global scales, which necessitate QR skills such as estimation, recognizing trends, and modeling future events. The project also focuses on moving student discourse from force dynamic (personal theory of the world) to true scientific discourse (science principles govern the system). Student's support of their views of environmental issues should include qualitative accounts based on data.

The quantitative reasoning research team for the project focuses on the QR aspects of how environmental literacy develops. An extensive literature review on QR in science, which is the focus of this paper, was conducted to support the development of the three environmental science learning progressions and related assessments and teaching experiments. As we explored the extensive and complex connections between environmental literacy and QR, a framework arose that incorporated elements of QR in the literature which were essential to those connections. This framework includes four components: (1) the Quantification Act, (2) Quantitative Literacy, (3) Quantitative Interpretation, and (4) Quantitative Modeling. The structure of the QR framework was derived from the literature review; extensive work with science and mathematics teachers through a professional development project called QR STEM which focused on interdisciplinary STEM teaching of the interplay of energy and environment; and interviews of middle and high school students on QR for the Culturally Relevant Ecology project. The literature review, framework, and interviews are the first steps in the creation of a hypothesized learning progression for $Q R$ in environmental science. The goal for the $\mathrm{QR}$ framework and $\mathrm{QR}$ learning progression is to interface with the environmental science learning progressions (e.g., the learning progression on the carbon cycle and storage). The QR learning progression will be reported in a future article. Our focus in this paper is the framework.

\section{Working Definition of Quantitative Reasoning}

The ability to think quantitatively is essential for citizens of a democracy, for it allows them to make informed decisions at home, in the workplace, and on complicated national and international issues that impact their local communities. QR is a complicated construct with many names and definitions, including: numeracy, number sense, deductive reasoning, mathematical literacy, quantitative literacy, problem solving, contextualized mathematics, mathematical modeling, and quantitative reasoning. So it is important for us to clarify how we are 
defining quantitative reasoning in our work. Representative definitions of quantitative reasoning and the related concept of quantitative literacy are provided in Table 1. These definitions have some common threads, such as the use of mathematics and statistics within a context and sophisticated reasoning with elementary mathematics. They also have significant differences. The first two definitions emphasize basic mathematics being used in sophisticated ways; others include more on habit of mind. For some, the focus is on use in making personal decisions, while others broaden this to making citizenship decisions about global issues.

Table 1

Definitions of Quantitative Reasoning

Steen (2004)

Langkamp and Hull (2007)

International Life Skills

Survey (ILSS 2000)

Programme for International Student Assessment (PISA 2000).

Hollins University (2011)

National Numeracy Network (NNN 2011)

BYU (2011)

Kolata (1997).

Bennett and Briggs (2008)
Quantitative literacy involves sophisticated reasoning with elementary mathematics more than elementary reasoning with sophisticated mathematics

Environmental problems can be better understood using number sense, basic algebra, simple models, and introductory statistics. Quantitative reasoning requires elementary mathematical concepts and techniques used in sophisticated ways

Quantitative literacy is an aggregate of skills, knowledge, beliefs, dispositions, habits of mind, communication capabilities, and problem-solving skills that people need in order to engage effectively in quantitative situations arising in life and work

Mathematical literacy is an individual's capacity to identify and understand the role that mathematics plays in the world, to make well-founded mathematical judgments and to engage in mathematics in ways that meet the needs of that individual's current and future life as a constructive, concerned, and reflective citizen

Quantitative reasoning is the application of mathematical concepts and skills to solve real-world problems. In order to perform effectively as professionals and citizens, students must become competent in reading and using quantitative data, in understanding quantitative evidence and in applying basic quantitative skills to the solution of real-life problems

Quantitative reasoning is the power and habit of mind to search out quantitative information, critique it, reflect upon it, and apply it in public, personal and professional life

Numerical abilities that equip students with the capacity to understand and explain the world in quantitative terms; to interpret numerical data; and to evaluate arguments that rely on quantitative information and approaches

Beyond arithmetic and geometry, quantitative literacy also requires logic, data analysis, and probability. It enables individuals to analyze evidence, to read graphs, to understand logical arguments, to detect logical fallacies, to understand evidence, and to evaluate risks. Quantitative literacy means knowing how to reason and how to think

Quantitative literacy is the ability to interpret and reason with quantitative information, information that involves mathematical ideas or numbers. Quantitative reasoning is the process of interpreting and reasoning with quantitative information

We want our definition to account for the conceptions of the quantification act (Thompson 2011) and the processes of modeling and interpreting models (Duschl et al. 2007). Both conceptions are gaining prominence and should be 
given consideration as important components of quantitative reasoning. In the National Research Council Report, Taking Science to School, Duschl et al. (2007) make the call to move learning in the sciences towards literacy and modeling. That report specifically identifies four proficiencies in science that all students should attain:

Strand 1: Know, use, and interpret scientific explanations of the natural world.

Strand 2: Generate and evaluate scientific evidence and explanation.

Strand 3: Understand the nature and development of scientific knowledge.

Strand 4: Participate productively in scientific practices and discourse.

These four proficiencies support a move from science as inquiry to science practices rooted in model-building and model-refining. They move science out of its current silos of biology, chemistry, earth systems, and physics into a more integrated STEM (Science, Technology, Engineering, Mathematics) approach focused on the application of science in real-world contexts.

Despite the focus of quantitative reasoning on applying mathematical skills and analysis of data through statistical processes, QR is not the same as mathematics or statistics. In the seminal work Mathematics and Democracy: The case for quantitative literacy, Steen (2001, p. 5) states:

Quantitative literacy is more a habit of mind, an approach to problems that employs and enhances both statistics and mathematics. Unlike statistics, which is primarily about uncertainty, numeracy is often about the logic of certainty. Unlike mathematics, which is primarily about a Platonic realm of abstract structures, numeracy is often anchored in data derived from and attached to the empirical world.

The need for quantitatively literate citizens arose in the late twentieth century as numbers became the dominant form of acceptable evidence in socio-political arenas, exposing a public which lacks the appropriate QR skills (Steen 2001). Steen identifies components of quantitative literacy which citizens should acquire, including confidence with mathematics (numeracy, estimation), cultural appreciation of mathematics (nature and history), interpreting data, logical thinking, making decisions, using mathematics in context, number sense, practical computation skills, prerequisite knowledge of algebra, geometry, statistics, and symbol sense.

Shavelson (2008) seeks a definition of quantitative reasoning by exploring three approaches to the topic: psychometric (behavioral roots), cognitive (mental process roots), and situative (social-contextual roots). The psychometric tradition has reached a consensus that there is a QR factor; that is, performance on QR tests is distinguishable from performance on other mathematics tests. $\mathrm{QR}$ requires reasoning based on mathematical properties and relations, with a low demand on computation and high demand on reasoning with numbers, operations, and patterns. Shavelson found cognitive research employing interviews and the think- 
aloud technique which focused on what kinds of reasoning processes are brought to bear in responding to $\mathrm{QR}$ type tasks to be lacking. Situative researchers view QR within a community of practice (Shavelson 2008, p. 8):

...those individuals engaged in culturally relevant activities in which reasoning quantitatively is demanded and the various resources of the community would be brought to bear on those activities. They would view a person accomplished in QR as having the capacity to engage others in working together to think critically, reason analytically and to solve a problem, for example. Cognitive abilities, from this perspective, reside in a community of practice.

Madison (2006a) provides a situative definition of QR as carried out in reallife, authentic situations; its application is in the particular situation, one dependent upon context including socio-politics. QR problems are deeply contextualized, ill-defined, open-ended, real-world tasks that require analysis, critical thinking, estimation, interdisciplinary approaches, and the capacity to communicate a solution, decision, or course of action clearly in writing. His contrast of mathematics and QR is summarized in Table 2. The Mathematical Association of America's notion of QR (MAA 1998) includes interpreting models, using multiple representations (symbolic, visual, numeric, graphic), applying arithmetical, algebraic, geometric, and statistical methods, estimating to determine reasonableness, and recognizing limits of algorithmic methods.

Table 2

Contrast of Mathematics with QR

\begin{tabular}{ll} 
Mathematics & Quantitative Reasoning \\
Power in abstraction & Real, authentic contexts \\
Power in generality & Specific, particular applications \\
Some context dependency & Heavy context dependency \\
Society independent & Society dependent \\
Apolitical & Political \\
Methods and algorithms & Ad hoc methods \\
Well-defined problems & Ill-defined problems \\
Approximation & Estimation is critical \\
Heavily disciplinary & Interdisciplinary \\
Problem solutions & Problem descriptions \\
Few practice opportunities outside classroom & Many practice opportunities outside classroom \\
Predictable & Unpredictable \\
\hline
\end{tabular}

To this point we have focused on quantitative reasoning within a context other than mathematics, so some clarification may be needed. In fact, Table 2 
may be interpreted as falsely suggesting $\mathrm{QR}$ is something separate from mathematics or that it is not a vital part of mathematical thinking and reasoning for mathematicians. We interpret the table as laying out the difference between mathematics done abstractly without a real-world context versus mathematics driven by a context of importance to an individual. This does not predicate that QR is outside the domain of mathematics or that all mathematics is well-defined or predictable. Surely the quantification act is essentially mathematical in nature. Without this mathematical act, quantitative reasoning does not occur. So we do not argue that QR cannot occur without a real-world context; rather, we want to focus intently on quantitative reasoning within context and, in this article, within the context of science. We want to explore the intensely interdisciplinary STEM nature of $\mathrm{QR}$ as an integrating factor when used within context. We believe that it is within real-world context that $\mathrm{QR}$ transcends both mathematics and the context.

From our reading of the literature cited above as well as our own work focused on QR in STEM, we define quantitative reasoning within context (QRC) as follows for the purpose of building a $\mathrm{QR}$ framework for a $\mathrm{QR}$ learning progression to interface with environmental science.

Quantitative Reasoning in Context (QRC) is mathematics and statistics applied in real-life, authentic situations that impact an individual's life as a constructive, concerned, and reflective citizen. QRC problems are context-dependent, interdisciplinary, open-ended tasks that require critical thinking and the capacity to communicate a course of action.

For the remainder of this paper we will refer to quantitative reasoning in context (QRC) simply as quantitative reasoning or $\mathrm{QR}$.

\section{QR Framework}

We propose a quantitative reasoning framework that has four key components:

1. Quantification Act (QA): Mathematical process of conceptualizing an object and an attribute of it so that the attribute has a unit measure.

2. Quantitative Literacy (QL): Use of fundamental mathematical concepts in sophisticated ways for the purpose of describing, comparing, manipulating, and drawing conclusions from variables developed in the quantification act.

3. Quantitative Interpretation (QI): Ability to use models to discover trends and make predictions.

4. Quantitative Modeling (QM): Ability to create representations to explain a phenomenon and to revise them based on fit to reality. 
These components interact within a quantitative reasoning cycle (Fig. 1). When individuals reflect upon a real-life authentic situation that impacts their communities or their personal lives, they will likely begin reasoning about the situation using a qualitative science account of the phenomena. The qualitative account may be based only on personal discourse (personal experiential theory of the world), rise to the level of including a school science discourse (acquired knowledge of science often without deep understanding), and perhaps progress to a full scientific discourse (science principles explaining the phenomena). Even at the full scientific-discourse level, some individuals may not have engaged in quantitative reasoning; in fact they may have actively avoided using QR. A quantitative account should be sought to provide data-driven support for the qualitative account and to provide evidence supporting conclusions.

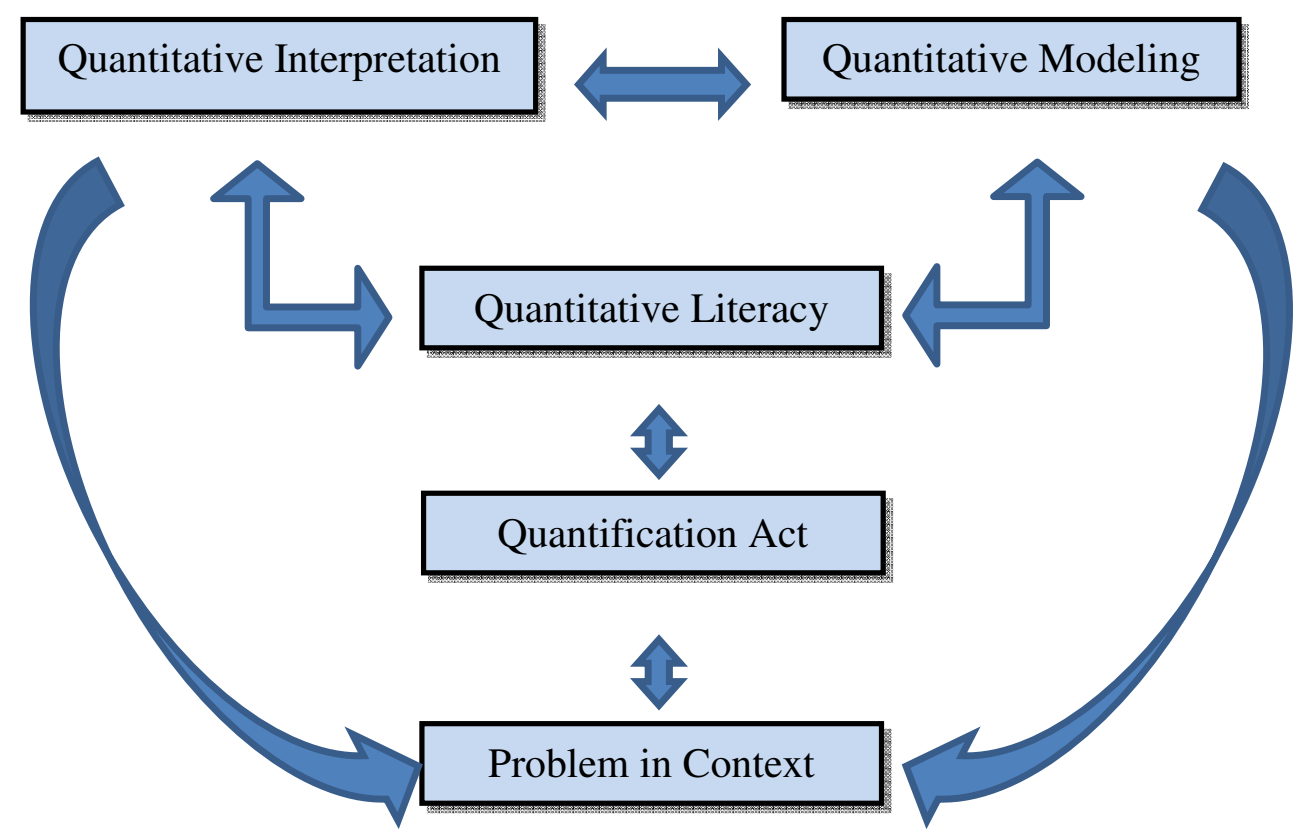

Figure 1. Quantitative Reasoning Cycle: QA, QL, QI and QM.

\section{Quantification Act}

The quantitative reasoning cycle begins with the individual engaging in the quantification act by identifying objects, observing their attributes, and assigning measures. Quantification provides variables that can be operated on mathematically or statistically. One must have a level of quantitative literacy to 
manipulate, compare, and relate these variables. The variables may appear in a mathematical representation such as a graph or equation, requiring one to engage in quantitative interpretation. Finally one might relate and combine the variables and thus engage in quantitative modeling. These four components of quantitative reasoning are laid out in Table 3. The table is not exhaustive; for example, it does not include geometric analysis, but lists what we have seen arise as mathematical and statistical tools of import in the context of environmental science. The output of QR is often a model that is then applied within the context of the situation to answer a question. The model may be a loosely connected set of relationships, a table, a graph, a science systems model, or even an analytical mathematical function. This model will need to be tested against the real-world situation and probably modified, leading back to the need to further quantify. Thus the cycle repeats.

Table 3

\begin{tabular}{|c|c|c|c|}
\hline Quantification Act & Quantitative Literacy & $\begin{array}{l}\text { Quantitative } \\
\text { Interpretation }\end{array}$ & Quantitative Modeling \\
\hline Variable Identification & $\begin{array}{l}\text { Numeracy } \\
\end{array}$ & Representations & Logic \\
\hline Attribute & Small/large & Graphs/diagrams & Problem Solving \\
\hline Measure & $\begin{array}{c}\text { Numbers } \\
\text { Scientific Notation }\end{array}$ & $\begin{array}{l}\text { Equations } \\
\text { Linear }\end{array}$ & Problem Formulation \\
\hline Communication & Logic & Quadratic & Modeling \\
\hline Force-dynamic & & Power & Normal Distribution \\
\hline Scientific discourse & Measurement & Exponential & Regression Model \\
\hline \multirow[t]{2}{*}{ Quantitative discourse } & Accuracy & Statistical displays & linear \\
\hline & Precision & Translation & polynomial \\
\hline Context & Estimation & & power \\
\hline Avoids QR & Units & Science diagrams & exponential \\
\hline Computation Driven & & Complex systems & logarithmic \\
\hline \multirow[t]{2}{*}{ Situative view } & Proportional Reasoning & & Logistic Growth Model \\
\hline & Fraction & Statistics \& Probability & Multivariate Model \\
\hline Variation & Ratio & Randomness & Simulation Model \\
\hline Causation & Percent & Evaluating Risks & Scientific Diagram \\
\hline Correlation & Rates/Change & Normal Distribution & Table \& Graph Models \\
\hline \multirow[t]{8}{*}{ Covariation } & Proportions & Statistical Plots & \\
\hline & Dimensional & Correlation & Inference \\
\hline & Analysis & Causality & Inference \\
\hline & & Z-scores & Hypothesis Testing \\
\hline & Basic Prob/Stats & Confidence Intervals & Practical Significance \\
\hline & Empirical Prob. & & \\
\hline & Counting & Logarithmic Scales & \\
\hline & Central tendency & & \\
\hline
\end{tabular}

\section{Quantitative Literacy}

Quantitative Literacy (QL) underlies both the interpretation and building of models. The variables resulting from quantification are operated on through $\mathrm{QL}$ 
and include such basics as performing computations, comparing, and estimating. QL is mostly arithmetic in nature, epitomizing the sophisticated use of basic mathematics. Within the quantitative literacy component we have identified four major elements (Table 3) that underpin the sciences: numeracy, measurement, proportional reasoning, and descriptive statistics and basic probability.

There is a great deal of variation in definitions of numeracy, from a mastery of arithmetic symbols and processes to being equivalent with quantitative literacy. We define numeracy as the ability to reason with numbers. Numeracy then is the logic and problem-solving aspect of $\mathrm{QR}$ on the arithmetic level. Numeracy includes having number sense, mastery of arithmetic processes (addition, subtraction, multiplication, and division), logic and reasoning with numbers, orders of magnitude, weights and measures.

Number sense is defined as awareness and understanding about what numbers are, their relationships, their magnitude, the relative effect of operating on numbers, including the use of mental mathematics and estimation (Fennel and Landis 1994). Number sense includes the concepts of magnitude, ranking, comparison, measurement, rounding, degree of accuracy, and estimation.

Measurement is of central importance to science, so it is separated out from numeracy in the framework as a second element of quantitative literacy which includes accuracy, precision, estimation, and measurement units.

Proportional reasoning is often a major conceptual barrier to students, inhibiting their ability to reason quantitatively in science. Here we include preproportional reasoning skills such as an understanding of fraction, ratio, percent, and rates, all leading up to proportions.

Basic probability and descriptive statistics are essential in data analysis in the sciences and require mostly arithmetic processes, so they are included as a fourth quantitative literacy element.

\section{Quantitative Interpretation}

Quantitative Interpretation (QI) expands upon the use of arithmetic skills in QL to include more mathematically sophisticated algebraic, geometric, statistical, and calculus processes. The QL focus on discovering relationships between variables is supplanted in QI by interpreting models to explore trends and make predictions, a skill that is essential for science literacy. We will use the term model in a broader sense than the typical mathematical interpretation of model as a function representing a situation. Models for us include any representation of data and data relationships which allows for interpreting a distinct case within context, exploring trends, drawing inferences, and making predictions.

QI entails interpreting models represented as tables, graphs, statistical graphical displays, equations, and complex scientific diagrams, as well as being able to translate between models. It includes the ability to interpret data using 
probability (randomness, evaluating risks) and statistical analysis (normal distributions, correlation, causality, $z$-scores, confidence intervals). Algebraic techniques such as logarithms are included under QI since they are common in science and provide a means of interpreting very small or very large scales.

QI at the most basic level is the ability to interpret the relationship between two variables; to interpret a model at a given instance or point. At the intermediate level it entails being able to identify trends, to interpret change. At the upper level it is the ability to make predictions through interpolation and extrapolation, to see correlations between data sets, to explain covariation between two variables, and to determine not only the direction but strength of association.

\section{Quantitative Modeling}

Quantitative Modeling (QM) is inexorably interconnected with quantitative interpretation, for surely when we create models we do so to interpret them. But QM extends QI by requiring the individual to create the model rather than interpret one that is given.

We define QM as the act of model creation or model generation. At the same time, we acknowledge that constructed thinking about or with a model is an essential process in model building. We assign this act of constructed thinking about or with models to QI. It could be argued that a non-scientist will not often create a model, but extending existing models to answer new questions and understanding how models are created are essential to quantitative reasoning.

QM requires a high level of reasoning, including logical thinking, problem solving, hypothesis testing, and caution in making over generalizations. QM engages individuals in formulating problems, developing linear, power, exponential, multivariate, and simulation models, and creating table, graph and even scientific diagram models. QM includes statistical hypothesis testing and understanding practical significance. Duschl's view of science as model building and model testing is underpinned by the ability to quantitatively model a phenomenon.

\section{Research Grounding of the QR Framework}

In the previous section, we gave an overview of quantitative reasoning in context and identified the four components of a framework for it. In this section we ground this framework in the literature.

\section{Quantification Act}

An underlying cognitive attribute of $\mathrm{QR}$ is the process of quantification, which underpins QL, QI, and QM. Quantification is known to be a significant 
component in modeling and has been found to be difficult for students (Thompson 2011).

Thompson and Saldanha (2003) provide an example of quantification as a root of quantitative reasoning by considering the question: What is torque and how might one quantify it? According to Thompson and Saldanha (2003), the "object" one conceives of as torque is a system involving turning something around a fixed point that behaves differently the farther from the fixed point you are. The attribute of torque is the "amount of twist," as in the case of recognizing it takes more strength to hold a pail of water farther from our body then closer. The measure of torque associated with this attribute is more complicated, since the measure must take into account simultaneously the distance that a force is applied from the fixed end, the amount of force being applied, and that amount of "twistiness" is proportional to each of these components.

Quantification is the conceptualization process in which quantities are assigned to attributes, with properties and relationships formed among them. Thompson (2011) provides evidence of quantification within a science context with an 8th grade class of students by addressing how they might measure the explosiveness of a grain silo. Collectively the students were quantifying the problem by first thinking that an explosion occurs when flames burn fast along with other knowledge they recalled from science regarding oxidation. The students then started to discuss volume of grain dust in relation to surface area exposed, eventually concluding that they need a unit measure of dust surface area per dust volume per silo volume as a way to measure explosiveness. This process required students to quantify the problem by conceptualizing attributes and how they would measure them (Thompson 2011).

Part of the conceptualization process of the quantification act is the ability to conceive of the problem mentally through an image. A study conducted by Moore, Carlson and Oehrtman (2009) shows the necessity of a correct mental image in order to quantify the problem and create relationships between the attributes. The study conducted with pre-calculus students shows that when students undergo the process of quantification, they create mental images using drawings or physical objects to represent a given problem. Once students were able to create the correct image, they were able to create correct formulas for solving a given problem. On the other hand, without this mental model, the students found no meaning in the formulas (Moore et al. 2009).This mental image is just as important for a teacher. "Project Pathways: Opening Routes to Math and Science Success for all Students,", which examined the professional development of secondary mathematics teachers, clearly showed the importance of teachers having a productive image of good student quantitative reasoning, including an

\footnotetext{
${ }^{2}$ NSF DUE 0412537, Marilyn Carlson, PI
} 
image of how their actions can influence student thinking (Thompson, pers. comm., 2012).

The process of quantification does not focus on a numerical solution, but rather on the conceptual process of solving a given problem. Smith and Thompson (2007) give examples of this process by comparing a numerical solution to a given problem with a quantitative or conceptual solution. Consider the following problem: A father will be 38 years old at some point when he will also be 3 times as old as his daughter, who is currently 7 years old. How old is he right now? The act of quantification takes problems such as this and emphasizes mathematical reasoning when solving problems by focusing on quantities and relationships among them (Smith and Thompson 2007). In this case, the difference between the two people's ages and the ratio of how many times older the father is than the daughter would be the relationships of focus. Having created these quantities and their relationships, students are able to develop a conceptual understanding of the given problem and in turn use mathematical concepts to solve the problem. These conceptual ideas create support for using algebra as a tool for problem solving.

The ability to quantify the problem is necessary if students are to find meaning in numerical computations rather than memorizing formulas which they never derived. According to Madison, "Many students do not believe that mathematics has very much to do with their everyday lives" (Madison 2006b, p. 2325). Madison (2006b) presents a pedagogical challenge: students have been taught for many years in traditional mathematics classrooms so their habits and attitudes can become obstacles. Thompson (1994) and Lobato and Siebert (2002) showed that teaching students formulas, such as slope is rise over run or speed is distance divided by time, does not provide students with the understanding to apply these concepts. Another difficulty is that in order for students to accept that they should understand a concept they must be engaged. In this case they should be engaged in the material to a greater extent than in a traditional mathematics classroom (Dingman and Madison 2010; Madison 2006b). Some positive outcomes have arisen in the studies done by Dingman and Madison. They concluded, "One of the positive changes we have seen is the modest shift in the students' views regarding the relevance of the mathematics in their everyday life. By placing the mathematical and statistical topics in real-world contexts, the connections to their life are much more real and apparent than their past experiences in learning mathematics" (Dingman and Madison 2010, p. 6).

An important aspect of quantification is covariational reasoning, which is defined as "cognitive activities involved in coordinating two varying quantities while attending to the ways in which they change in relation to each other" (Carlson et al. 2002, p. 354). Lobato and Siebert (2002) conducted a teaching experiment in a course on linear functions and slopes in which they closely 
studied the progress of one particular student. The focus was on a wheelchair ramp problem where the students were questioned about the steepness of the ramp. In particular, Lobato and Siebert believed that focusing on slope as a calculation to determine steepness would cause some loss in potential transfer of knowledge; rather, the focus should be on slope as a ratio of two varying quantities measuring some attribute; that is on covariation. The student first believed that height was the only determining factor of steepness. After some probing from the interviewer, the student was able to accept that length also influenced the steepness of the ramp, but the student could not vary the height and length of the ramp independently. However, by the end of the teaching experiment, the student was able to construct a ratio between the height and length. This is an example of the development of covarying quantities. It is important to take note that when the student was asked if he had learned about slope, he responded that it is rise over run; however, he did not interpret slope to be a measure of steepness of the ramp. This indicates that the attributes of the object must first be determined and a relationship formed between the attributes, before measurement is performed.

A case study by Thompson (1994) also shows the trouble students have in understanding proportional reasoning or ratio is due to a lack of understanding of covariational reasoning. The student in Thompson's study thought of time as an implicit quantity with respect to distance, in a given speed problem. Just as in the Lobato and Siebert study, the student was not able to see two quantities of equal stature in the beginning of the teaching experiment. However, in both cases, the students were able to create the ratio by the end of the experiment by first acknowledging two explicit quantities. In this case, the student acknowledged time as an explicit quantity and in turn created the ratio. The traditional teaching of speed as distance divided by time does not allow for the development of the concept of speed as a ratio and has little relevance to the understanding of speed (Thompson 1994).

\section{Quantitative Literacy}

We propose four elements of QL related to science: numeracy, measurement, proportional reasoning, and descriptive statistics/basic probability (Table 3).

Numeracy includes the ability to work with very large numbers (U.S. energy consumption of $100,000,000,000,000,000,000$ joules) and very small numbers (diameter of hydrogen nucleus is 0.000000000000001 meter). Issues of order of magnitude as powers of 10 represented through scientific notation become paramount. Science is replete with extremely small and large numbers which are often incomprehensible to students. Three techniques for bringing numbers into perspective are estimation, comparisons, and scaling (Bennett and Briggs 2008). 
Numeracy is the key to understanding data in our current society. Understanding requires more than formulas; rather, it requires the ability to reason and think quantitatively (Steen 2001). Wilkins extends the definition of QL to include people who are willing to "engage in situations that require a functional level of quantitative reasoning" (Wilkins 2000, p. 408). Unfortunately, many students lack QL skills due to a shallow coverage of these concepts in schools, due to a focus on college prep and a narrow curriculum with the singular goal of calculus as the culminating experience. Many of the fundamental QL skills such as measurement, geometry, data analysis and probability do not get much time in the curriculum (Madison 2003).

The sciences require careful comprehensive measurement of quantities such as distance, area, volume, discharge, mass, density, force, pressure, work, moment, energy, power, and heat. Some quantities are measured directly (e.g., length in meters or feet, weight in Newtons or pounds, and temperature in Celsius, Fahrenheit, or Kelvin) using a variety of tools such as rulers, scales, inclinometers, spectrometers, and fluorometers, while others are measured indirectly or are calculated from other measures (e.g., area, volume, stream discharge [volume/time], density [mass/volume], force [mass times acceleration], pressure [force/area], work [force times distance], and power [work/time]). Fundamental characteristics of measure are accuracy (how close the measurement is to the actual value), precision (how refined the measure is), and error (Langkamp and Hull 2007).

Wiser and Smith (2009) conjecture a learning progression in which students move from a conception of felt weight to quantifying weight as measured by a scale. This progression requires understanding the quantitative nature of measure. They propose that students move from measuring weight and volume to an understanding of density by graphing weight as a function of volume.

A study by Jones, Taylor, and Broadwell (2009) was done on the sense of scale and estimation using the body as a measurement tool. Using the body as a ruler allowed students to better visualize a measure and become more accurate in estimation. Consequently, students who had a better understanding of proportional reasoning also performed better by giving more accurate values of estimation.

Proportional reasoning is positively correlated with applying scale and estimation in real-world problems. So lack of proportional reasoning can be an obstacle for students in their understanding of concepts in science. Students' sense of scale was also studied by Delgado et al. (2007), who examined students' understanding of how big one object was compared to another, and the object's absolute size.

Proportional reasoning encompasses complex cognitive abilities which include both mathematical and psychological dimensions. It requires a significant 
conceptual shift from concrete operational to formal operational levels of thought (Piaget and Beth 1966). It has been proposed as a major barrier to students' development of mathematical understanding, as well as negatively impacting the development of scientific understanding. Its pivotal position in science is as the most common form of structural similarity, a critical aspect of recognizing similar patterns in two different contexts. In addition, proportional reasoning underpins many of the QL elements, including measurement, numeracy, and dimensional analysis.

Proportional reasoning is a "form of mathematical reasoning that involves a sense of co-variation and of multiple comparisons, and the ability to mentally store and process several pieces of information" (Lesh et al. 1988). Lesh et al. (1988) consider the essential characteristic of proportional reasoning to involve reasoning about the holistic relationship between two rational expressions (fractions, quotients, rates, and ratios). Proportional reasoning is not the ability to employ the cross multiplication algorithm; in fact rote use of this algorithm often replaces such reasoning. Early phases in proportional reasoning involve additive reasoning $(A-B=C-D)$ and multiplicative reasoning $(A \times B=C \times D)$. Traditional proportional reasoning involves relationships of the type $A / B=C$ / $D$, where one of the values is unknown. Karplus et al. (1983) views proportional reasoning as a linear relationship between variables such as $y=m x$, where the $y$-intercept is 0 .

Proportional reasoning requires students to first understand fraction $a / b$, which at the most basic level is interpreted by students as comparing the part (numerator $a$ ) to the whole (denominator $b$ ) for like quantities. This basic concept of fraction underpins one notion of percentage as comparing part-to-whole. This is also an example of using a percentage to describe change.

Taylor and Jones (2009) conducted a study with middle school students on their proportional reasoning abilities. The students attended a summer camp in which they participated in activities on surface-area-to-volume applications. As a result, Taylor and Jones found that there is a significant correlation between proportional reasoning abilities and surface-area-to-volume relationships. The study shows that there may also be a relationship between proportional reasoning and the scaling concept in science.

Probability is essential in science; however, it is experimental probability that is the focus, not theoretical probability. Earth systems cannot be manipulated like dice to determine a theoretical probability. Often scientists can only estimate the probability through observations of the system. Empirical (experimental) probability is determining a probability based on observations or experiments.

Descriptive statistics allow us to summarize and describe data. The fundamental descriptive statistics are measures of the center of a distribution and measures of the spread in a distribution. Measures of central tendency include the 
mean, median, and mode; however, the mode is not often used in analyzing scientific data. The mean and median values for a data set can differ significantly, so it does make a difference what measure of central tendency is reported.

Intrinsically tied to measures of central tendency is the concept of variation, which measures how much the data are spread out. The simplest measure of variation is the range which, while easy to calculate, can be misleading, because one outlier can make it appear the data set is more spread than it is. To avoid this one can use quartiles (values that divide the data set into quarters) and the fivenumber summary - lowest value, lower quartile, median, upper quartile, and highest value. The most commonly used measure of variation is standard deviation. By Chebyshev's Rule, for any set of data at least $75 \%$ of the data lie within two standard deviations of the mean and at least $89 \%$ of the data lie within three standard deviations of the mean. Any data value that lies three or more standard deviations from the mean is commonly called an outlier, and it is common practice to discard them from the data set.

Most of what we have discussed to this point as elements of QL belong in the realm of number and arithmetic; only variation requires algebraic operations of taking roots or powers. So we see the meaning of Steen's admonition that QL is sophisticated reasoning with elementary mathematics. Other basic statistics that are used in science cross over from arithmetic to algebra such as $z$-scores (number of standard deviations a data point lies above or below the mean) and confidence intervals. But it is amazing what people can do with arithmetic if they can but reason with it within a context.

The fundamental mathematical concepts necessary for non-calculus-track students are addressed by Briggs (2004). Based on a study he conducted on a group of non-calculus-track undergraduate students fulfilling general education requirements, Briggs found that the fundamental mathematical knowledge needed were logical thinking skills, estimation, statistical literacy, and financial mathematical knowledge. Students find it difficult to attain these skills as they often do not see the connection between the mathematical knowledge they acquire and applications to their daily lives. QL must be made compelling to students by showing them examples of how it impacts their lives, which are filled with quantitative information (Briggs 2004).

\section{Quantitative Interpretation}

For QI we suggest four main elements: representations, science diagrams, statistics and probability, and logarithmic scales (Table 3). QI is the ability to use models to make predictions and determine trends.

Due to the fact that a model can take various forms (e.g., tables, graphs/diagrams, equations, statistical displays, or complex scientific diagrams), issues can arise with the translation between models representing the same 
content. For example, given a table and a graph of the same data, students may struggle to see the relationship between the two different representations. Understanding the multitude of representations available is important for organizing, synthesizing, explaining, and displaying data, which in turn is essential for being a citizen scientist.

The media, workplace, and our everyday lives have been filled with quantitative data. It is imperative that everyone be able to interpret and use the data presented to them to make informed decisions (Madison and Steen 2003; Steen 2001, 2004). According to the Quantitative Literacy Design Team, "Most U.S. students leave high school with quantitative skills far below what they need to live well in today's society (Steen 2001, p. 1)." Data from the Third International Mathematics and Science Study (TIMMS) revealed that students in the U.S. performed relatively low compared to other countries in their mathematical skills. However, the same students responded that they enjoy mathematics and are confident in their performance (Wilkins 2000).

As the American Education Reach Out organization states: "Representations are necessary to students' understanding of mathematical concepts and relationships" (AERO 2010, p. 11). Early understanding of multiple representations is important for students to progress mathematically (Schwartz and Martin 2004; Zahner and Corter 2010). Zahner and Corter (2010) propose in their model that students pass through four stages when problem solving. Stage 2 is mathematical problem representation. According to their model, to reach level 3 and 4, students must pass through this stage first. Therefore, the inability to interpret and represent a problem could be a barrier to student problem solving.

Representations take on numerous forms, from graphs and tables to equations and written text. They also vary in popularity. For example, Lowrie and Diezmann (2009) found maps are a type of representation that has increased in popularity recently. Maps are one representation that requires a certain amount of "decoding" (Logan and Greenlees 2008; Lowrie and Diezmann 2009), which can be very challenging for students. They argue that students can encounter difficulties when trying to separate graphical features from other demands posed by the task, such as linguistic knowledge and mathematical knowledge.

Examining multiple representations is important when discussing learning because often graphical representations and text appear side-by-side. Stroud and Schwartz (2010) base their study of metaphoric graphics in chemistry instruction on the notion of the redundancy effect. This occurs when students become overwhelmed with the amount of information presented as "text-based content" and it interferes with student learning (Stroud and Schwartz 2010). Thus, knowing how students read, interpret, and process simultaneous representations when learning content is important to consider when developing learning progressions and planning instruction (Clement et al. 1981). This supports our 
inclusion of the ability to translate between different representations. When more than one representation is presented side-by-side, it is important for the student to make meaning of each representation and draw connections among the different representations.

When asked to classify mathematical problems according to their difficulty, many would argue that story problems are among the most difficult for students to solve. According to a study conducted by Koedinger and Nathan (2004), the exact opposite seems to be true. They divide the problem-solving process into two phases, a comprehension and a solution phase (Koedinger and Nathan 2004). During the comprehension phase the type of problem representation chosen plays a vital role for the students' understanding as well as their problem-solving strategies. Koedinger and Nathan (2004) discovered in their study that students showed fewer difficulties concerning story word problems than with symbolic problems, such as equations, when the language and context used are accessible to them.

Friel, Curcio, and Bright (2001) investigated the comprehension of statistical graphs. They identified four categories influencing graph comprehension: purpose for using a graph, characteristics of the tasks, characteristics of the discipline, and the characteristics of graph readers. According to Friel et.al. (2001), a vital component of graph comprehension is understanding that there are three areas of graph perception, namely "visual decoding, judgment task, and context" (Friel et al. 2001, p. 152). All three components need to be adequately addressed to ensure improved graph comprehension. This is also supported by the study conducted by Zahner and Corter (2010). They call for further research to enhance teachers' understanding of how students comprehend graphs, which in turn can result in new instructional strategies.

Thompson has conducted multiple studies on representations (Oehrtman et al. 2008; Thompson 1994, 1999; Thompson and Saldanha 2000). One aspect Thompson (1994) focused on was the concept of average rate and speed. Students display difficulties when it comes to distinguishing ratio and rate. Thompson (1994, 53) exclaims that "any problem typology suffers this same deficiency, namely that any given situation can be conceived in a multitude of ways." During his teaching experiment he discovered that a model can easily be interpreted or viewed in different ways, which emphasizes the subjective character of interpretation.

Thompson and Saldanha (2000) also studied the role of representations in the field of statistics and probability. They found that student misunderstandings in this area arise as early as elementary school and can be reduced through exposing students more to statistics and probability in early years of school. Gibson and colleagues (2011) investigated the effect of using statistical representations taken from case studies and news reports on a group of undergraduates with varying 
quantitative reasoning skills. Students with greater ability in arithmetic skills achieved more accurate results on a QR computer-based test. The test provided students with articles from which they were asked to extract and assess the numerical information in terms of the underlying content, and give estimations of, for example, ratios. They also found that students with lower-ability arithmetic skills are "more attentive to, and affectively more engaged by personalized information provided by detailed case reports" (Gibson et al. 2011, p. 114).

\section{Quantitative Modeling}

Quantitative modeling is the ability to create representations to explain phenomena. We propose four elements for QM: logic, problem solving, modeling, and inference. QM requires a large amount of logical thinking and reasoning for an individual to produce a model. Just as QI refers to a multitude of representations, QM has many different forms as well (e.g., formulating problems; developing linear, power, exponential, multivariate, and simulation models; creating table, graph and scientific diagram models).

Science as model-building is defined in terms of learning science as a process of building theories and models using evidence, checking them for internal consistency and coherence, and testing them empirically (Duschl et al. 2007). The NRC also states in A Framework for K-12 Science Education: Practices, Crosscutting Concepts, and Core Ideas (NRC 2011) that STEM disciplines permeate our lives and thus are central to meeting humanity's most pressing challenges. Common to both national reports is a focus on the interdisciplinary study of real-world problems that emphasize key STEM understandings and practices. An important component of this integration is quantitative reasoning, which can serve as an integrating factor of mathematics and statistics into science.

The seminal research done by Schwarz et al. (2009) in the Modeling Designs for Learning Science (MoDeLS) project defines scientific modeling as elements of practice including constructing, using, evaluating, and revising scientific models, and the metaknowledge that guides and motivates the practice. Their learning progression for scientific modeling has two dimensions: the use of scientific models as tools for predicting and explaining, and the idea that models change as understanding improves. While MoDeLS provides a scientific qualitative account of modeling, we believe it needs to be expanded to include the quantitative account of modeling across grades 6-12.

Lesh (2006) calls for a deep understanding of complex systems which are becoming increasingly prominent in the 21 st century. This pertains to nearly any attempt at modeling real-world phenomena. Students need to be more familiar with how to focus on the most important processes they want to model, as well as strategies of modeling. According to Lesh (2006), mathematics includes the learning of sets of rules to the same degree as it includes the ability to model real- 
world situations. This idea of enhancing the design of tasks that engage students in complex modeling is exemplified in a study by Lesh et al. (2008). They introduce the idea of model-eliciting activities (MEA) to enhance students' modeling abilities.

Thompson's (2011) approach to QM focuses on the quantification act, especially concerning dynamic situations, and quantitative covariation. He defines two aspects that are essential when using mathematics to model dynamic situations. First, students need to understand the quantities themselves and visualize that their images include values that vary. Second, students need to form a representation of the "object made by uniting those quantities in thought and maintaining that unit while also maintaining a dynamic image of the situation in which it is embedded" (Thompson 2011, p. 27).

QM plays a major role in the sciences (Lehrer et al. 2000; Matthews 2007; Svoboda and Passmore 2011; Adúriz-Bravo 2012), and modeling in science needs to be taught in a dynamic manner. Scientists develop, use, and revise their models in a cyclic process. This process should be accessible for students so they understand the dynamic nature of science. Matthews (2007) describes the process as beginning with observations of real objects, which then need to be conveyed linguistically in some way. Within this step is where quantification has to take place. This conveyed information, or model, is set within a discourse and can now be scientifically debated. The final step is the revision of the model and then the cycle starts over again. Adúriz-Bravo (2012) describes this process with the words: "inventing, applying, refining, and learning models (Adúriz-Bravo 2012, p. 16).

Lehrer et al. (2000) investigated two situations where children learn through design in elementary school. Most important for education by design are the tasks, tools, and representations. The basic idea is to align classroom activities to how scientists in the real world work. It begins with a problem (task) which needs to be specified and should lead to the construction of a working model. This model needs to be tested and tried and if needed re-designed. Finally, students need to elaborate the important ideas behind their model and data. Their two classroom implementations also demonstrated how important it is to connect new material to children's existing knowledge.

Doerr and English (2003) investigated how instructional tasks can enhance students' modeling. Their instructional tasks were different from traditional textbook problems and addressed "the creation of ranked quantities, operations and transformations on those ranks, and, finally, the generation of relationships between and among quantities to define descriptive and explanatory relationships" (Doerr and English 2003, p. 131). They provided instruction which differed from the traditional way of simply guiding students through specific problem-solving strategies, enabling them to develop their own ways of 
approaching, refining, and expanding their thinking about problems. Their results showed that after students were exposed to the novel instruction, they were capable of defining their own quantities (ranks) and operating on those quantities to build their basis for interpreting and revising their models. Additionally, they discovered that since students approached problems with a variety of different models, the communication about and translations between models was facilitated.

Lehrer and Schauble (2004) also discovered the need for improving instructional strategies. Their study explored upper elementary students' thinking in the context of natural distribution. Students worked with data to model plant growth. The main emphasis during instruction was placed on investigations carried out by the students. The students developed different models in order to draw inferences about plant growth as well as make predictions for the future. This study demonstrated how important it is for students to construct their own models of real-world phenomena, allowing them to draw inferences and to reach a deeper understanding.

One of the most difficult types of modeling for students is algebraic modeling. In a case study conducted by Izsák (2003) on how students go about modeling a physical device, he discovered that students can develop a set of skills that allows them to construct, evaluate and test their developed equations. This set of skills results from a combination of students' prior common knowledge and carefully guided student-based instruction. The students were able to develop a linear system of equations, evaluate it by plugging in values, and discuss and revise it in collaboration with each other. Hence, algebraic modeling can be mastered by students with the appropriate guidance, but it is not common in current classroom practice.

To instantiate science as modeling in the classroom we must move from direct instruction of STEM as a collection of facts to be mastered and from a narrow hypothesis-testing view of scientific inquiry, toward curriculum, instruction and assessment models that embrace the four proficiencies strands in Taking Science to School. Science teaching would be driven by science as modelbuilding and refinement. This reformulation of curriculum, instruction and assessment proposes a significant change in the current teaching and learning of STEM which is within the purview of the schools. It will require a significant shift in both student and teacher expectations in the classroom. Science from a model-building perspective is best achieved through integrated, interdisciplinary STEM instruction that incorporates place-based (Smith and Sobel 2010) and problem-based pedagogies (Edelson and Reiser 2006). Engaging students in realworld problems will require them to bring to bear knowledge and understandings from multiple subject areas, including biology, chemistry, earth systems science, physics, mathematics, and statistics. Building models and testing them will push 
both the teachers' and students' capabilities. There is a need to study the potential for students to engage in model-building and testing, establishing trajectories of student development though the creation of learning progressions which can assist teachers in tracking students' formative development, and eventually to the construction of professional development programs. The theoretical foundations and pathways so established will guide the creation of developmentally appropriate performance tasks that will provide students with experiences that further their understanding of key concepts across STEM.

\section{Discussion}

There are other QR frameworks, so what makes ours (Table 3) unique? We will address this by comparing and contrasting it with the Quantitative Literacy VALUE Rubric (Rhodes 2010) developed by the Association of American Colleges and Universities (AAC\&U 2010). The VALUE rubrics were developed by teams of college and university faculty examining existing campus QL rubrics and related documents.

The AAC\&U definition of quantitative literacy includes $Q R$ and numeracy.

Quantitative Literacy (QL) - also known as Numeracy or Quantitative Reasoning (QR) is a "habit of mind," competency, and comfort in working with numerical data. Individuals with strong QL skills possess the ability to reason and solve quantitative problems from a wide array of authentic contexts and everyday life situations. They understand and can create sophisticated arguments supported by quantitative evidence, and they can clearly communicate those arguments in a variety of formats (using words, tables, graphs, mathematical equations, etc., as appropriate). (AAC\&U 2010)

AAC\&U (2010) also identifies six components of QL:

1. Interpretation: Ability to explain information presented in mathematical forms.

2. Representation: Ability to convert relevant information into various mathematical forms.

3. Calculation: Ability to calculate to solve a problem.

4. Application/Analysis: Ability to make judgments and draw appropriate conclusions based on the quantitative analysis of data, while recognizing the limits of this analysis.

5. Assumptions: Ability to make and evaluate important assumptions in estimation, modeling, and data analysis.

6. Communication: Expressing quantitative evidence in support of argument or purpose of the work. 
Our QR framework includes calculation (VALUE's component 3) as part of QL, but focuses on QL as the sophisticated use of basic mathematics that is frequently used in the sciences. We see these as the skills that a quantitatively literate citizen should be able to bring to the problem. QI in our framework includes interpretation (component 1) and representation (component 2), which are essential in deciphering information provided in graphs, tables, and science models. QM in our framework includes application/analysis (component 4) and assumptions (component 5), but our focus is on building, testing and refining models as a way of learning science. Here we highlight the essential aspect of students constructing models as not just interpreting models they are given. Finally, our QA component includes elements of communication (component 6); however, we build on the research on quantification to accentuate the extremely critical role that quantification plays in students moving from science context to mathematical representation and back to science context.

\section{Concluding Remarks}

The QR framework presented in this paper (Table 3) was developed to inform quantitative aspects of the environmental literacy learning progressions created by the Culturally Relevant Ecology, Leaning Progressions, and Environmental Literacy project. The current research in science learning progressions and on quantification, as well as the focus on modeling in the new Common Core Mathematics Standards and Framework for K-12 Science Education, led us to include the components of quantification act, quantitative interpretation, and quantitative modeling, alongside the essential quantitative literacy component. The resulting $\mathrm{QR}$ framework created interest within the project in hypothesizing a learning progression for QR in Science.

Creating learning progressions is an iterative research process that involves development of assessments and extensive analysis of interviews. We are early in the development of the QR progression, and so we do not present it here. To date we have conducted over 50 interviews with $6^{\text {th }}$ to $12^{\text {th }}$ grade students to assess the $\mathrm{QR}$ progression and the QR framework which underpins it. Thus far we have found the QR framework to be a useful construct for this work.

\section{Acknowledgments}

We would like to acknowledge the detailed review and comments provided by members of the WISDOM ${ }^{\mathrm{e}}$ Quantitative Reasoning Team, especially Dr. Larry Hatfield of the University of Wyoming and Dr. Patrick Thompson of Arizona State University. We also wish to acknowledge comments by Dr. Richard Duschl of Penn State University. Finally the extensive work by the core Pathways QR Team was essential to the production of this article, including Jennifer 
Schuttlefield of the University of Wisconsin Oshkosh, Jennifer Forrester of the University of Wyoming, and Nissa Yestness of the University of Northern Colorado.

\section{References}

AAC\&U (American Association of Colleges and Universities). 2010. VALUE: Valid Assessment of Learning in Undergraduate Education.

http://www.aacu.org/value/rubrics/index_p.cfm

Adúriz-Bravo, A. 2012. A 'semantic' view of scientific models for science education. Science and Education 21: 1-19. http://dx.doi.org/10.1007/ s11191-011-9431-7 (accessed February 6, 2012)

AERO (American Education Reach Out). 2010. AERO Mathematics curriculum framework: K-8 standards and performance indicators.

http://www.projectaero.org/aero standards/mathematics-framework/AEROMathematicsCurriculumFramework.pdf (accessed February 3, 2012)

Bennett, J., and W.L. Briggs. 2008. Using and understanding mathematics: A quantitative reasoning approach. $4^{\text {th }}$ ed. Reading, MA: Addison.

Briggs, W.L. 2004. What mathematics should all college students know? In Current practices in quantitative literacy, ed. R. Gillman, 17-19. Washington, DC: Mathematical Association of America.

BYU (Bringham Young University), 2011. Aims of a BYU education. http://aims.byu.edu/p/aims (accessed February 3, 2012)

Carlson, M., S. Jacobs, E. Coe, S. Larsen, and E. Hsu. 2002. Applying covariational reasoning while modeling dynamic events: A framework and a study. Journal for Research in Mathematics Education 33: 352-378. http://dx.doi.org/10.2307/4149958 (accessed February 6, 2012)

Clement, J., J. Lochhead, and G.S. Monk. 1981. Translation difficulties in learning mathematics. The American Mathematical Monthly 88: 286-290. http://dx.doi.org/10.2307/2320560

Corcoran, T., F. A. Mosher, and A. Rogat. 2009. Learning progressions in science: An evidence-based aproach to reform. Philadelphia, PA: Consortium Policy Research in Education.

Delgado, C., S. Y. Stevens, N. Shin, M. Yunker and J. Krajcik. 2007. The Development of Students' Conception of Size. Paper presented at the National Association for Research in Science Teaching Conference, New Orleans, LA.

Dingman, S.W., and B. L. Madison. 2010. Quantitative reasoning in the contemporary world, 1: The course and its challenges. Numeracy 3(2): Article 4. http://dx.doi.org/10.5038/1936-4660.3.2.4 (accessed February 10, 2012)

Doerr, H., and L. English. 2003. A modeling perspective on students' mathematical reasoning about data. Journal for Research in Mathematics Education 34(2): 110136. http://dx.doi.org/10.2307/30034902 (accessed February 6, 2012) 
Duschl, R.A., H. A.Schweingruber, and A. W. Shouse. 2007. Taking science to school: Learning and teaching science in grades $K-8$. Washington, DC: National Academies Press.

Edelson, D.C., and B. J. Reiser. 2006. Making authentic practices accessible to learners: Design challenges and strategies in. In The cambridge handbook of the learning sciences, ed. K. Sawyer, 335-354. Cambridge, PA: Cambridge University Press.

Fennell, F., and T.E. Landis. 1994. Number sense and operation sense. In Windows of opportunity: Mathematics for students with special needs, eds. C.A. Thornton and N.S. Bley, 187-203. Reston, VA: National Council of Teachers of Mathematics.

Friel, S., F. Curcio, and G. Bright. 2001. Making sense of graphs: Critical factors influencing comprehension and instructional implications. Journal for Research in Mathematics Education 32:124-158. http://dx.doi.org/10.2307/749671 (accessed February 10, 2012)

Gibson, R., C. Callison, and D. Zillmann. 2011. Quantitative literacy and affective reactivity in processing statistical information and case histories in the news. Media Psychology 14: 96-120. http://dx.doi.org/10.1080/15213269.2010.547830 (accessed February 10, 2012)

Hollins University. (2011). Course goals and criteria document. http://www.hollins.edu/registrar/documents/ESP goalscriteria.pdf (accessed February 3, 2012)

ILSS (International Life Skills Survey). 2000. Policy Research Initiative. Statistics. Canada.

Izsák, A. 2003. "We want a statement that is always true": Criteria for good algebraic representations and the development of modeling knowledge. Journal for Research in Mathematics Education 34: 191-227. http://dx.doi.org/10.2307/30034778 (accessed February 6, 2012)

Jones, G., A. Taylor, and B. Broadwell. 2009. Estimating linear size and scale: Body rulers. International Journal of Science Education 31: 1495-1509. http://dx.doi.org/10.1080/09500690802101976 (accessed February 15, 2012)

Karplus, R., S. Pulos., and E. K. Stage. 1983. Early adolescents' proportional reasoning on rate problems. Educational Studies in Mathematics 14: 219-233. http://dx.doi.org/10.1007/BF00410539 (accessed February 6, 2012)

Koedinger, K., and M. Nathan. 2004. The real story behind story problems: Effects of representations on quantitative reasoning. The Journal of the Learning Sciences 13: 129-164. http://dx.doi.org/10.1207/s15327809jls1302_1 (accessed February 10, 2012)

Kolata, G. 1997. "Understanding the news.” In Why numbers count: Quantitative literacy for tomorrow's America, ed. L. A. Steen. New York, NY: The College Board.

Langkamp, G., and J. Hull. 2007. Quantitative reasoning and the environment. Upper Saddle River, NJ: Pearson Education.

Lehrer, R., and L. Schauble. 2004. Modeling natural variation through distribution. American Educational Research Journal 41: 635-679. http://dx.doi.org/10.3102/00028312041003635 (accessed February 12, 2012)

S. Carpenter, and D. Penner. 2000. The interrelated development of inscriptions and conceptual understanding. In Symbolizing and communicating in mathematics 
classrooms: Perspectives on discourse, tools, and instructional design, eds. P. Cobb, E. Yackel, and K. McClain, 325-360. Mahwah, NJ: Lawrence Erlbaum Associates.

Lesh, R. 2006. Modeling students' modeling abilities: The teaching and learning of complex systems in education. The Journal of the Learning Sciences 15: 45-52. http://dx.doi.org/10.1207/s15327809j1s1501_6 (accessed February 15, 2012)

— T. Post, and M. Behr. 1988. Proportional Reasoning. In Number concepts and operations in the middle grades, eds. J. Hiebert and M. Behr, 93-118. Reston, VA: Lawrence Erlbaum \& National Council of Teachers of Mathematics.

Lesh, R., J. Middleton, E. Caylor, and S. Gupta. 2008. A science need: Designing tasks to engage students in modeling complex data. Educational Studies in Mathematics 68: 113-130. http://dx.doi.org/10.1007/s10649-008-9118-4 (accessed February 12, 2012)

Lobato, J., and D. Siebert. 2002. Quantitative reasoning in a reconceived view of transfer. Journal of Mathematical Behavior 21: 87-116. http://dx.doi.org/10.1016/S07323123(02)00105-0 (accessed February 6, 2012)

Logan, T., and J. Greenlees. 2008. Standardised assessment in mathematics: The tale of two items. In Navigating currents and charting directions: Proceedings of the $31^{\text {st }}$ annual conference of the Mathematics Education Research Group of Australia, eds. M. Goos, R. Brown, and K. Makar, 655-658. Brisbane: Mathematics Education Research Group of Australia.

Lowrie, T., and C. M. Diezmann 2009. National numeracy tests: A graphic tells a thousand words. Australian Journal of Education 53: 141-158. http://www.csu.edu.au/research/glim/downloads/AJE.pdf (accessed February 6, 2012)

MAA (Mathematics Association of America). 1998. Quantitative reasoning for college graduates: A complement to the standards. http://www.maa.org/past/ql/ql_toc.html

Madison, B.L. 2003. Articulation and quantitative literacy: A view from inside mathematics. In Why numeracy matters for schools and colleges, eds. B. L. Madison, and L. A. Steen, 153-164. Mathematical Association of America. . 2006a. Assessment and QL: double trouble. Keynote address presented at the Northeast Consortium on Quantitative Literacy's annual meeting, Amherst College._. 2006b. Pedagogical challenges of quantitative literacy.

Proceedings of the Joint Statistical Meetings, 2323-2328. Alexandria, VA: American Statistical Association.

— schools and colleges. Princeton, NJ: National Council on Education and the Disciplines.

Matthews, M. 2007. Models in science and in science education: An Introduction. Science and Education, 16: 647-652. http://dx.doi.org/10.1007/s11191-007-9089-3 (accessed February 6, 2012)

Moore, K. C., M. P. Carlson, and M. Oehrtman. 2009. The role of quantitative reasoning in solving applied precalculus problems. Conference on Research in Undergraduate Mathematics Education (CRUME). Raleigh, NC. 
NNN (National Numeracy Network). 2011. What is numeracy/QL/QR?

http://serc.carleton.edu/nnn/resources/index.html (accessed February 3, 2012)

NRC (National Research Council). 2011. A Framework for K-12 Science Education:

Practices, Crosscutting Concepts, and Core Ideas. Washington, DC: National

Academy Press.

Oehrtman, M. C., M. P. Carlson, and P. W. Thompson. 2008. Foundational reasoning abilities that promote coherence in students' understandings of function. In Making the connection: Research and practice in undergraduate mathematics, eds. M. P. Carlson \& C. Rasmussen, 27-42. Washington, DC: Mathematical Association of America. http://dx.doi.org/10.5948/UPO9780883859759.004

Piaget, J., and E. Beth. 1966. Mathematical epistemology and psychology. Dordrecht, The Netherlands: D. Reidel.

PISA (Programme for International Student Assessment). 2000. Organization for Economic Cooperation and Development (OECD).

Rhodes, T, ed. 2010. Assessing Outcomes and Improving Achievement: Tips and Tools for Using Rubrics. Washington, DC: Association of American Colleges and Universities.

Schwartz, D. L., and T. Martin. 2004. Inventing to prepare for future learning: The hidden efficiency of encouraging original student production in statistics instruction. Cognition and Instruction 22: 129-184. http://dx.doi.org/10.1207/s1532690xci2202_1

Schwarz, C.V., B.J. Reiser, E. A. Davis, L. Kenyon, A. Archer, D. Fortus, Y. Shwartz, B. Hug, and J. Krajcik. 2009. Developing a learning progression for scientific modeling: Making scientific modeling accessible and meaningful for learners. Journal of Research in Science Teaching 46: 632-654. http://dx.doi.org/10.1002/tea.20311

Shavelson, R. 2008. Reflections on quantitative reasoning: An Assessment Perspective. In Calculation vs. context: Quantitative literacy and its implications for teacher education, eds. B. L. Madison, and L. A. Steen, 22-47. Washington, DC: Mathematical Association of America.

Smith, G.A., and D. Sobel. 2010. Place-and community-based education in schools (Sociocultural, Political, and Historical Studies in Education). New York: Routledge.

Smith III, J., and P. W. Thompson. 2007. Quantitative reasoning and the development of algebraic reasoning. In Algebra in the Early Grades, eds. J.J. Kaput, D.W. Carraher, and M.L. Blanton, 95-132. New York, NY: Lawrence Erlbaum Associates.

Steen, L. A. 2001. Mathematics and democracy: The case for quantitative literacy, ed. L. A. Steen, Princeton, NJ: The Woodrow Wilson National Fellowship Foundation, The National Council on Education and the Disciplines.

- 2004. Achieving quantitative literacy: an urgent challenge for higher education. Washington, DC: Mathematical Association of America.

Stroud, M. J., and N. H. Schwartz. 2010. Summoning prior knowledge through metaphorical graphics: An example in chemistry instruction. Journal of Educational Research 103: 351-366. http://dx.doi.org/10.1080/00220670903383077 (accessed February 12, 2012) 
Svoboda, J., and C. Passmore. 2011. The strategies of modeling in biology education. Science and Education 1-24. doi: 10.1007/s11191-011-9425-5 (accessed February 20, 2012)

Taylor, A. R., and M. G. Jones. 2009. Proportional reasoning ability and concepts of scale: Surface area to volume relationships in science. International Journal of Science Education 31: 1231-1247. http://dx.doi.org/10.1080/09500690802017545 (accessed February 15, 2012)

Thompson, P. W. 1994. The development of the concept of speed and its relationship to the concepts of rate. In The development of multiplicative reasoning in the learning of mathematics, eds. G.Harel and J. Confrey, 181-234. Albany, NY: SUNY Press.

. 1999. Some remarks on conventions and representations. Panel for the PME-NA XXI Working Group on Representations. Cuernavaca, Mexico.

- 2011. Quantitative reasoning and mathematical modeling. In New perspectives and directions for collaborative research in mathematics education, eds. L. L. Hatfield, S. Chamberlain, and S. Belbase, 33-57. Laramie, WY: University of Wyoming.

, and L. Saldanha. 2000. Conceptual issues in understanding sampling distributions and margins of error. Proceedings of the Twenty-second Annual Meeting of the International Group for the Psychology of Mathematics Education. Tucson, Arizona.

- 2003. Fractions and multiplicative reasoning. In Research companion to the Principles and Standards for School Mathematics, eds. J. Kilpatrick, G. Martin and D. Schifter, 95-114. Reston, VA: National Council of Teachers of Mathematics.

Wilkins, J. L. 2000. Preparing for the $21^{\text {st }}$ century: The status of quantitative literacy in the United States. School Science and Mathematics 100: 405-418. http://dx.doi.org/10.1111/j.1949-8594.2000.tb17329.x (accessed February 6, 2012)

Wiser, M., and C. Smith. 2009. How does cognitive development inform the choice of core ideas in the physical sciences? Expert Meeting on Core Ideas in Science. Washington DC.

Zahner, D. and J. Corter. 2010. The process of probability problem solving: Use of external visual representations. Mathematical Thinking and Learning 12: 177-204. http://dx.doi.org/10.1080/10986061003654240 (accessed February 12, 2012) 